\title{
Estudio del Fenómeno Explosión de Superficie en la Reacción NO+CO en Pt(100) por Simulación de Monte Carlo Dinámico
}

\author{
Salomón de J. Alas y Luis Vicente \\ Universidad Nacional Autónoma de México, Facultad de Química, Departamento de Física y \\ Química Teórica, 04510 México, D.F.-México \\ (e-mail: espectros7@gmail.com, luis.vicente@servidor.unam.mx)
}

\section{Resumen}

Se presenta el estudio de la reducción catalítica de NO por CO en una superficie de Pt(100) por medio de una simulación de Monte Carlo dinámico. El objetivo es analizar la evolución temporal y espacio-temporal del sistema. Considerando un mecanismo de reacción tipo Langmuir-Hinshelwood, se construye un modelo donde se involucran todos los pasos elementales de la reacción y se simula la llamada explosión de superficie. El análisis también considera recientes evidencias experimentales concernientes a la formación y decaimiento de una especie intermediaria (N-NO)* como el paso de reacción principal en la producción de $\mathrm{N}_{2}$. Los resultados de la simulación están de acuerdo con resultados experimentales de espectroscopia de desorción de masas. Además, la simulación permite correlacionar los máximos de producción de $\mathrm{N}_{2}$ y $\mathrm{CO}_{2}$ con la formación de patrones espaciales tipo célula sobre la superficie.

\section{Study of the Surface Explosion Phenomenon in the NO+CO Reaction on Pt(100) by Dynamic Monte Carlo Simulation}

\begin{abstract}
In this work the catalytic reduction of $\mathrm{NO}$ by $\mathrm{CO}$ on a $\mathrm{Pt}(100)$ surface by dynamic Monte Carlo simulation is presented. The objective is to analyze the temporal and spatial-temporal behavior. A Langmuir-Hinshelwood mechanism and a model where all the reaction steps are taken into account are considered and the so-called surface explosion is simulated. The analysis also includes recent experimental evidences concerning the formation and decay of a (N-NO) intermediary species which turns out to be important for the $\mathrm{N}_{2}$ formation. These simulation results show good quantitative agreement with experimental results obtained from isothermal mass spectroscopy desorption. Furthermore, the simulations allow correlating the maximum production of $\mathrm{N}_{2}$ with the formation of cellular patterns on the surface.
\end{abstract}

Keywords: dynamic Monte Carlo, NO reduction, surface explosion, cellular patterns 


\section{INTRODUCCION}

La reducción catalítica del NO ha sido objeto de muchos estudios debido a su relevancia en los estudios de mejoramiento de la calidad del aire. Los motores diesel, las plantas eléctricas y los hornos son las principales fuentes de emisión de $\mathrm{NO}$; representando el $90 \%$ de los compuestos $\mathrm{NO}_{\mathrm{x}}$. Los vehículos automotores cuentan con convertidores catalíticos para la reducción de emisiones contaminantes, las cuales representan entre 0.05 y $0.15 \%$ en volumen (Egelhoff, 1982). Además, esta reacción en condiciones alejadas del equilibrio termodinámico presenta una gran variedad de fenómenos no lineales interesantes como oscilaciones cinéticas (Imbihl y Ertl, 1995; Siera et al., 1991; Fink et al., 1991a), las cuales consisten en cambios periódicos en el tiempo de las concentraciones de las especies adsorbidas y formadas, formación de frentes de reacción-difusión, propagación de ondas químicas (o propagación de un frente de concentración) y la formación de patrones espacio-temporales (espirales y franjas). También se han observado fenómenos de histéresis y biestabilidad cuando se varían los parámetros de control externos de la reacción (presiones parciales, velocidades de flujo y temperatura).

El cambio periódico que experimenta la estructura del catalizador causa oscilaciones en la reacción de reducción de $\mathrm{NO}$, por diferentes agentes como $\mathrm{CO}, \mathrm{H}_{2}$ y $\mathrm{NH}_{3}$, por ejemplo, en el monocristal de $\mathrm{Pt}(100)$, el estado estable cuasi-hexagonal (hex) puede ser reestructurado hacia un estado metaestable $1 \times 1$ por efectos de adsorción de las moléculas, y puede reconstruir de manera inversa $1 \times 1 \rightarrow$ hex por la desorción de los productos (Imbihl y Ertl, 1995; Lombardo et al., 1993; Fink et al., 1991b). Otro mecanismo que promueve la formación de oscilaciones, en esta reacción, se debe a la gran interacción que existe entre las especies adsorbidas (Slinko et al., 1992). En particular, para la reacción NO+CO en Pt(100), a condiciones de $\mathrm{T}>430 \mathrm{~K}$ estos son los mecanismos aceptados y se han realizado diferentes estudios de Monte Carlo (MC) para explicar la aparición de los diferentes fenómenos no lineales (Zhdanov, 2002; Perera y Vicente, 2002).

A temperaturas bajas $(T<430 \mathrm{~K})$, la formación de oscilaciones se debe a una reacción autocatalítica que incrementa el número de sitios vacantes, lo cual favorece la disociación del NO sobre la superficie de $\operatorname{Pt}(100)$ (Makeev y Nieuwenhuys, 1998). La autocatálisis en la disociación de NO se manifiesta por la ocurrencia de picos muy estrechos (explosión de superficie) durante la producción de $\mathrm{N}_{2}$ a T $\sim 400 \mathrm{~K}$ observados por estudios de TPR (Temperature Programmed Reaction).

El objetivo del presente trabajo es estudiar la evolución cinética del carácter explosivo de la reacción $\mathrm{NO}+\mathrm{CO}$ en $\mathrm{Pt}(100)$ tomando en cuenta los mecanismos reportados en la literatura, en especial se considera la producción de $N_{2}$ por dos vías: a través de la combinación de $N_{(a d s)}+N_{(a d s)}$ y uno nuevo que toma en cuenta la formación de una especie intermediaria (N-NO)*, producida por la combinación $\mathrm{NO}_{(\text {ads })}+\mathrm{N}_{(\text {ads) }}$, la cual ha sido observada en recientes experimentos en la reacción $\mathrm{NO}+\mathrm{CO}$ sobre $\mathrm{Rh}(111)$ (Avalos et al., 2005; 2006). Este paso alterno de reacción para el sistema $\mathrm{NO}+\mathrm{CO} / \mathrm{Pt}(100)$ no ha sido descartado en observaciones experimentales (Ohno et al., 2003), además, los argumentos a favor de incluir tal paso se ven reforzados por cálculos de DFT (Neurock et al., 2004), inclusive, ya ha sido considerado en estudios de simulación (Alas y Zgrablich, 2006) en donde se analizó el comportamiento oscilatorio. Para llevar a cabo el presente estudio se utiliza una técnica de DMC, donde los parámetros utilizados en la simulación corresponden a los reportados experimentalmente, lo que ha permitido comparar directamente con resultados de Espectroscopía de Desorción de Masas (Lesley y Schmidt, 1985). El aporte de este estudio es que la técnica permite analizar la distribución de las especies sobre la superficie del catalizador y la formación de estructuras espaciales.

\section{MODELO Y METODO DE SIMULACION}

En el modelo propuesto la superficie del cristal Pt(100) está representada por una malla regular en 2$\mathrm{D}$ con condiciones de frontera periódicas y solamente se considera la fase $1 \times 1$. La malla consiste de $\mathrm{L} \times \mathrm{L}$ sitios; un sitio indica un átomo de Pt en la superficie y tiene cuatro vecinos. En las simulaciones se utilizan mallas de $1024 \times 1024$ sitios. La fase gaseosa es una mezcla de moléculas de NO y CO con presiones parciales $p_{\mathrm{NO}}$ y $p_{\mathrm{CO}}$, respectivamente. El esquema de reacción considera lo siguiente: 
a) formación de una especie intermediaria (N-NO)* como el paso principal en la producción de $\mathrm{N}_{2 \text { (gas), }}$

b) producción de $\mathrm{N}_{2 \text { (gas) }}$ vía el mecanismo clásico de recombinación $\mathrm{N}_{(\text {ads) }}+\mathrm{N}_{(\text {ads) }}$,

c) inhibición en la velocidad de disociación de $\mathrm{NO}_{(\mathrm{ads})}$ debido a la presencia de CO y $\mathrm{NO}$ adsorbidos como primeros vecinos (nn),

d) aumento en la velocidad de disociación de NO por la presencia de $\mathrm{N}_{(\mathrm{ads})}$ como $\mathrm{nn}$,

e) desorción de NO y CO adsorbidos, y

f) difusión superficial de las moléculas de NO y CO adsorbidos.

Se considera que la reacción $\mathrm{NO}+\mathrm{CO}$ en Pt(100) procede de acuerdo con el siguiente mecanismo tipo Langmuir-Hinshelwood:

$$
\begin{aligned}
& \mathrm{CO}_{(\text {gas })}+\text { sitio } \leftrightarrows \mathrm{CO}_{(\text {ads })} \\
& \mathrm{NO}_{(\text {gas })}+\text { sitio } \leftrightarrows \mathrm{NO}_{(\text {ads })} \\
& \mathrm{NO}_{(\text {ads })}+\text { sitio } \rightarrow \mathrm{N}_{(\text {ads })}+\mathrm{O}_{(\text {ads })} \\
& \mathrm{NO}_{(\text {ads })}+\mathrm{N}_{(\text {ads })} \rightarrow(\mathrm{N}-\mathrm{NO})^{\star}+\text { sitio } \\
& (\mathrm{N}-\mathrm{NO})^{\star} \rightarrow \mathrm{N}_{2(\text { gas })}+\mathrm{O}_{(\text {ads })} \\
& \mathrm{N}_{(\text {ads })}+\mathrm{N}_{(\text {ads })} \rightarrow \mathrm{N}_{2(\text { gas })}+2 \text { sitios } \\
& \mathrm{CO}_{(\text {ads })}+\mathrm{O}_{(\text {ads })} \rightarrow \mathrm{CO}_{2(\text { gas })}+2 \text { sitios }
\end{aligned}
$$

donde "sitio" denota sitios de adsorción en la superficie del catalizador de Pt(100); $\mathrm{NO}_{\text {(gas) }}$ y $\mathrm{CO}_{\text {(gas) }}$

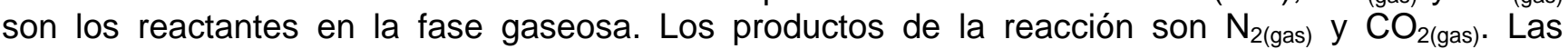
especies adsorbidas en las superficies se denotan con el subíndice ads. En la formación de $\mathrm{N}_{2 \text { (gas) }}$ se ha tomado en cuenta que el paso (6) es más lento que los pasos (4) y (5), como ha sido considerado por Avalos et al. (2005) y Alas y Zgrablich (2006), y ocurren con probabilidades de 0.3 y de 0.7 , respectivamente. La difusión de las especies se considera como saltos a sitios $n$ vacíos adyacentes.

En una simulación DMC la evolución temporal del sistema ocurre como un proceso estocástico de Markov y la distribución de probabilidad de los estados se describe por la ecuación maestra:

$$
\frac{\partial P_{\alpha}(t)}{\partial t}=\sum_{\beta}\left[W_{\beta \rightarrow \alpha} P_{\beta}(t)-W_{\alpha \rightarrow \beta} P_{\alpha}(t)\right]
$$

donde, $P_{\alpha}(t)$ y $P_{\beta}(t)$ son las probabilidades de encontrar al sistema en las configuraciones $\alpha$ y $\beta$, respectivamente, al tiempo $t$, y las $W^{\prime}$ s son las probabilidades de transición por unidad de tiempo de los diferentes procesos, indicados como subíndices.

Los pasos del algoritmo que se toman en cuenta durante la simulación de DMC son los siguientes:

(i) se selecciona un sitio de la superficie al azar con probabilidad $1 / N$; donde $N$ indica el numero total de sitios ocupados,

(ii) se escoge un paso de reacción $i$ al azar (por ejemplo, desorción, difusión, etc.) con probabilidad $W_{i} / R$, donde $R$ es la suma de las velocidades de todos los procesos posibles (con excepción de la adsorción), esto es, la velocidad de transición total del sistema,

(iii) si el paso de reacción $i$ seleccionado es viable sobre el sitio elegido, inmediatamente se ejecuta, y

(iv) después que cada sitio es seleccionado el tiempo se incrementa en $\Delta t$, de acuerdo con:

$\Delta t=-\frac{\ln \xi}{N R}$ 
donde $\xi$ es un número aleatorio seleccionado en el intervalo $(0,1)$. Esta ecuación da el tiempo real causado por una transición del sistema.

En la simulación se han tomado en cuenta todos los procesos (1-7). Los detalles se pueden encontrar en Alas y Vicente (2008), donde se simuló el espectro de TPR con precisión. Las constantes de velocidad para cada proceso se pueden calcular con los parámetros dados en la Tabla 1. En el caso de la disociación de NO, estos corresponden a valores de velocidades iniciales $\left(W_{\text {Noidis }}\right)$, pues este paso de reacción es afectado subsecuentemente debido a las partículas adsorbidas y sitios libres como nn.

Tabla 1: Valores de los parámetros usados en las simulaciones y de los experimentales. Los datos experimentales pueden ser consultados en Fink et al. (1991a, 1991b), donde, $p$ es la presión parcial, $S_{0}$ es el coeficiente inicial de adsorción, $v$ es el factor de frecuencia y $E_{a}$ es la energía de activación.

\begin{tabular}{|c|c|c|c|c|}
\hline Proceso & $p^{\exp }(\mathrm{Pa})$ & $p^{\text {sim }}(\mathrm{Pa})$ & $S_{0}^{\exp }$ & $\mathrm{S}_{0}^{\mathrm{sim}}$ \\
\hline adsorción CO & $3.0 \times 10^{-5}$ & $3.0 \times 10^{-5}$ & $\approx 0.8$ & 0.8 \\
\hline adsorción NO & $4.0 \times 10^{-5}$ & $4.0 \times 10^{-5}$ & $\approx 0.8$ & 0.8 \\
\hline & $v^{\exp }\left(s^{-1}\right)$ & $v^{\operatorname{sim}}\left(\mathrm{s}^{-1}\right)$ & $E_{\mathrm{a}}{ }^{\exp }(\mathrm{kJ} / \mathrm{mol})$ & $E_{\mathrm{a}}^{\mathrm{sim}}(\mathrm{kJ} / \mathrm{mol})$ \\
\hline desorción CO & $1.0 \times 10^{14}-10^{15}$ & $1.0 \times 10^{14}-10^{15}$ & $148.6-157.0$ & $148.6-157.0$ \\
\hline desorción NO & $1.7 \times 10^{14}-10^{15}$ & $1.7 \times 10^{14}-10^{15}$ & $142.3-154.9$ & $142.3-154.9$ \\
\hline disociación NO & $2.0 \times 10^{15}$ & $2.0 \times 10^{15}$ & 119.3 & 119.3 \\
\hline producción $\mathrm{N}_{2}$ & $1.3 \times 10^{11}$ & $1.3 \times 10^{11}$ & 84.6 & 84.6 \\
\hline producción $\mathrm{CO}_{2}$ & $2.0 \times 10^{8}$ & $2.0 \times 10^{8}$ & 58.6 & 58.6 \\
\hline difusión CO & -------- & $0-50$ & -------- & ------- \\
\hline difusión NO & -------- & $0-50$ & -------- & -------- \\
\hline
\end{tabular}

Otras suposiciones, de acuerdo al trabajo de Alas y Vicente (2008), que se consideran son: (i) no se toma en cuenta la producción de $\mathrm{N}_{2} \mathrm{O}$; (ii) las interacciones laterales entre las diferentes especies adsorbidas se consideran al modificar de manera apropiada la velocidad de disociación de NO; (iii) se desprecian la desorción y difusión de $\mathrm{O}_{(\text {ads) }}$; (iv) no se incluye la difusión de $\mathrm{N}_{(\text {ads) }}$.

\section{RESULTADOS Y DISCUSION}

Para realizar el análisis de la reacción NO+CO en función del tiempo se empieza con una superficie limpia de $\mathrm{Pt}(100)$ en la cual se lleva a cabo la adsorción de moléculas de CO y NO en la proporción $p_{\mathrm{CO}}: p_{\mathrm{NO}}=3: 4$. La temperatura y las presiones parciales se mantienen constantes durante todo el experimento simulado: $\mathrm{T}=400 \mathrm{~K}, p_{\mathrm{CO}}=3.0 \times 10^{-5} \mathrm{~Pa}$ y $p_{\mathrm{NO}}=4.0 \times 10^{-5} \mathrm{~Pa}$, siendo estos valores similares a los usados en los experimentos reales. Cuando la superficie se encuentra totalmente cubierta, esto es, $\theta=\theta_{\mathrm{CO}}+\theta_{\mathrm{NO}}=1$, se deja de adsorber $\mathrm{CO}$ y $\mathrm{NO}$ e inmediatamente se consideran los demás procesos (desorción, disociación, reacción entre las especies, etc.).

La Fig. 1 muestra las velocidades de producción de: (a) $\mathrm{CO}_{2 \text { (gas) }}$ y (b) $\mathrm{N}_{2 \text { (gas) }}$, medidas como cantidad de moléculas producidas por átomo de Pt por segundo (TON). Las curvas corresponden al promedio de 20 simulaciones. Estas gráficas muestran en particular el efecto de aumentar las velocidades de difusión, de $W_{\text {dif }}=10$ a $W_{\text {dif }}=30 \mathrm{~s}^{-1}$. A medida que se incrementa $W_{\text {dif }}$ los máximos de las curvas se recorren hacia tiempos menores (la explosión ocurre antes) y los semi-anchos de las curvas se reducen. Otro efecto observado es que se obtiene un valor límite en la posición del máximo, el cual se encuentra situado alrededor de $\mathrm{t}=18 \mathrm{~s}$.

Este efecto puede ser explicado de la siguiente manera: al principio de la simulación la superficie se encuentra llena de moléculas de $\mathrm{CO}_{(\text {ads })}$ y $\mathrm{NO}_{(\mathrm{ads})}$, en un momento dado el proceso de desorción crea sitios vacíos al azar, entonces las moléculas de $\mathrm{NO}_{(\text {ads })}$ pueden disociar en $\mathrm{N}_{(\text {ads })}$ y $\mathrm{O}_{\text {(ads) }}$, los cuales pueden empezar a reaccionar y generar más sitios vacíos, por tanto, se forma una reacción 
autocatalítica, produciendo de esta manera un frente de reacción. Al aumentar la velocidad de difusión también aumenta la probabilidad de que las especies se encuentren para reaccionar entre

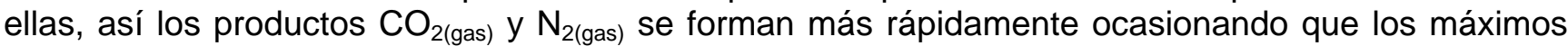
aparezcan a tiempos menores.
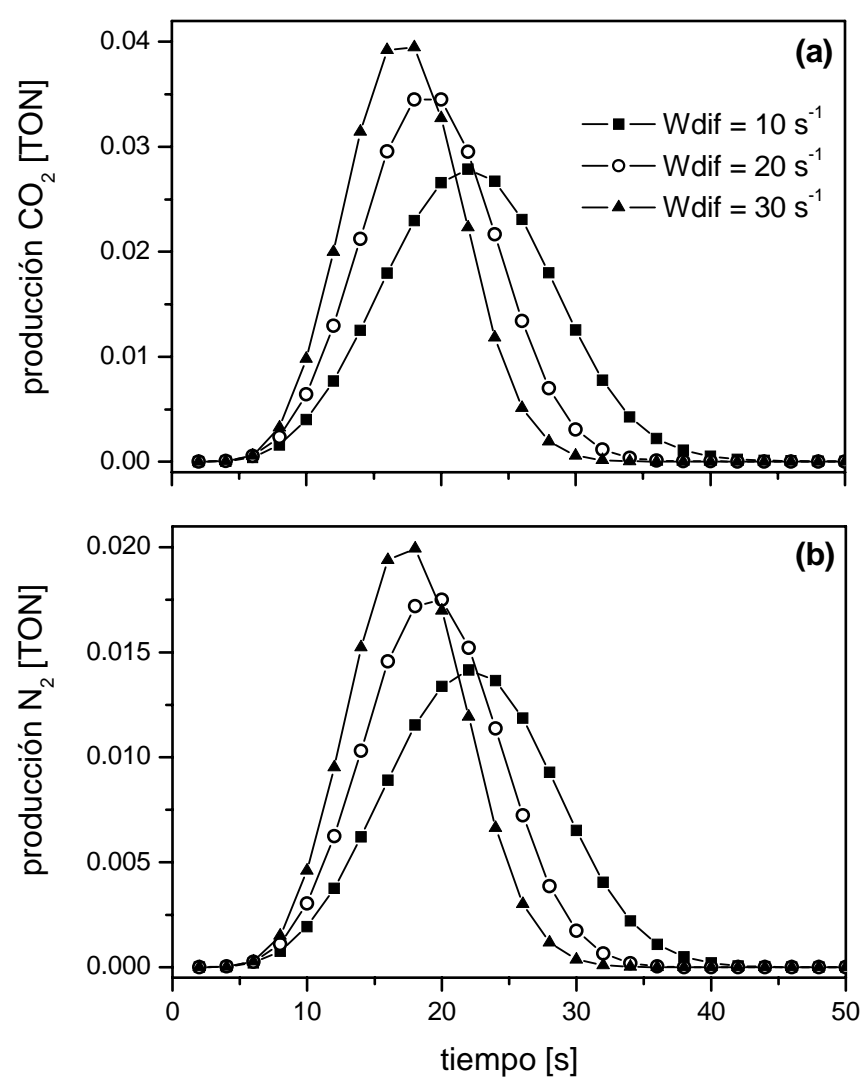

Fig. 1: Simulación del espectro de desorción: (a) $\mathrm{CO}_{2}$ y (b) $\mathrm{N}_{2}$. Se muestran diferentes valores de $W_{\text {dif }}=W_{\text {Codif }}=W_{\text {NOdif. }}$ Las otras velocidades son: $W_{\text {codes }}=3.1 \times 10^{-6} \mathrm{~s}^{-1} ; W_{\text {NOdes }}=1 \times 10^{-5} \mathrm{~s}^{-1}$; $W_{\text {NOidis }}=5.275 \mathrm{~s}^{-1}$. El tamaño de la malla es de $1024 \times 1024$ sitios.

Además, se ha analizado el efecto de incrementar las velocidades de desorción de $\mathrm{CO}_{(\text {ads })}$ y $\mathrm{NO}_{(\text {ads) }}$, pero manteniendo los parámetros dentro del rango de los valores experimentales de la Tabla 1.

La Fig. 2 muestra el resultado obtenido al variar los parámetros de desorción de CO: $v=1.0 \times 10^{15} \mathrm{~s}^{-1}$ y $E_{\mathrm{a}}=148.6 \mathrm{~kJ} / \mathrm{mol}$, y para la desorción de $\mathrm{NO}: v=1.7 \times 10^{15} \mathrm{~s}^{-1}$ y $E_{\mathrm{a}}=142.3 \mathrm{~kJ} / \mathrm{mol}$. Como se puede observar, comparados con la figura 1, los máximos y los anchos medios de las curvas de producción de $\mathrm{CO}_{2 \text { (gas) }}$ y $\mathrm{N}_{2 \text { (gas) }}$ se localizan a tiempos menores. Lo que es interesante ahora es que los picos se encuentran alrededor de 8 y 10 segundos y los anchos medios tienen un valor alrededor de 4 y 7 segundos.

La tabla 2 muestra un resumen acerca de las mediciones que se hicieron de los máximos y los anchos medios de la Fig. 2.

Estos resultados son completamente consistentes con las observaciones experimentales reportadas por Lesley y Schmidt (1985), en sus Espectros de Desorción de Masas. En efecto, durante la formación explosiva de $\mathrm{CO}_{2}$ y $\mathrm{N}_{2}$, reportan que los máximos ocurren alrededor de 10 a 30 segundos y que las curvas tienen un ancho medio de aproximadamente de 4 a 6 segundos. 

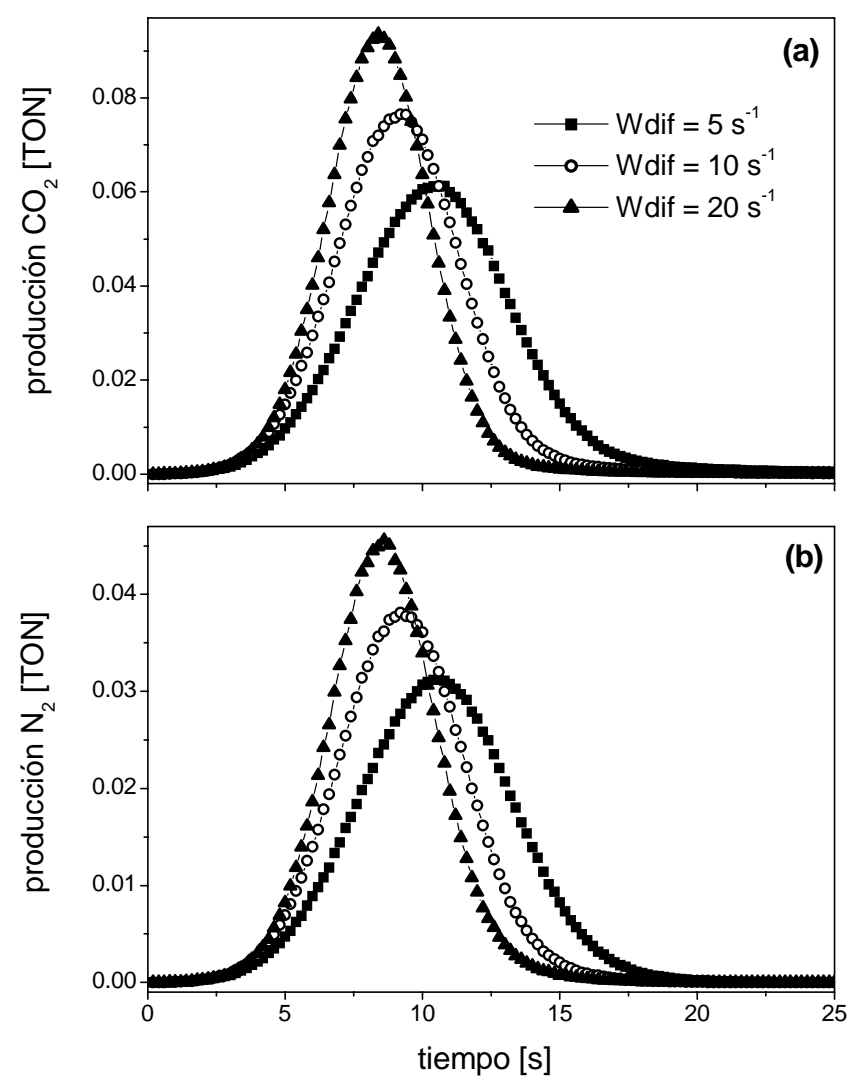

Fig. 2: Simulación del espectro de desorción. Se muestran diferentes valores de $W_{\text {dif }}=W_{\text {codif }}=W_{\text {NOdif }}$. Las otras velocidades son: $W_{\text {Codes }}=3.94 \times 10^{-5} \mathrm{~s}^{-1} ; W_{\text {NOdes }}=4.45 \times 10^{-4} \mathrm{~s}^{-1} ; W_{\text {Noidis }}=5.275 \mathrm{~s}^{-1}$. El tamaño de la malla es de $1024 \times 1024$ sitios.

Tabla 2: Medidas obtenidas de los máximos y anchos medios durante la producción de $\mathrm{CO}_{2 \text { (gas) }}$ y $\mathrm{N}_{\text {2(gas) }}$ para diferentes $W_{\text {dif. }}$

\begin{tabular}{|c|c|c|c|c|}
\cline { 2 - 5 } \multicolumn{1}{c|}{} & \multicolumn{2}{c|}{ producción $\mathrm{CO}_{2}(\mathrm{TON})$} & \multicolumn{2}{c|}{ producción $\mathrm{N}_{2}(\mathrm{TON})$} \\
\hline$W_{\text {dif }}\left(\mathrm{s}^{-1}\right)$ & máximos $(\mathrm{s})$ & anchos medios $(\mathrm{s})$ & máximos $(\mathrm{s})$ & anchos medios $(\mathrm{s})$ \\
\hline 5 & $\approx 10.4$ & $\approx 6.5$ & $\approx 10.4$ & $\approx 6.6$ \\
\hline 10 & $\approx 9.2$ & $\approx 5.3$ & $\approx 9.2$ & $\approx 5.3$ \\
\hline 20 & $\approx 8.4$ & $\approx 4.3$ & $\approx 8.6$ & $\approx 4.5$ \\
\hline
\end{tabular}

Finalmente, se ha podido observar, en las simulaciones, que durante la ocurrencia del fenómeno "explosivo" de la reacción se pueden crear diferentes frentes de reacción al azar en la superficie y se originan patrones espacio-temporales, en este caso, los llamados patrones celulares.

La Fig. 3 muestra los detalles de la creación y formación de frentes de reacción. La Fig. 3(a) indica la formación de frentes en diferentes sitios de la malla al tiempo t = $9 \mathrm{~s}$. En la Fig. 3(b) se observa como estos frentes empiezan a crecer y se expanden a través de la superficie, $t=12 \mathrm{~s}$. Las Figuras siguientes, 3(c), 3(d) y 3(e) muestran como los frentes chocan entre ellos, t = 17, 20 y $23 \mathrm{~s}$, respectivamente. La Fig. 3(e) corresponde al momento en el cual se alcanza el máximo del pico de desorción de la Fig. 1 con $W_{\text {dif }}=10 \mathrm{~s}^{-1}$. En la Fig. 3(f) se observa como los frentes se han extinguido casi completamente al tiempo $\mathrm{t}=27 \mathrm{~s}$, aquí se puede ver que permanecen algunos átomos de $\mathrm{N}_{(\mathrm{ads})}$ y $\mathrm{O}_{(\text {ads })}$ sin reaccionar sobre la superficie. 


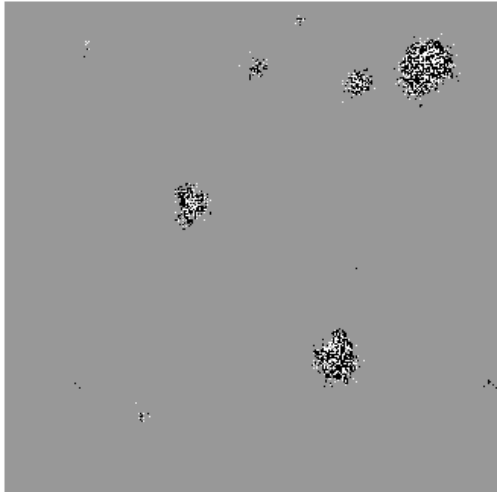

(a)

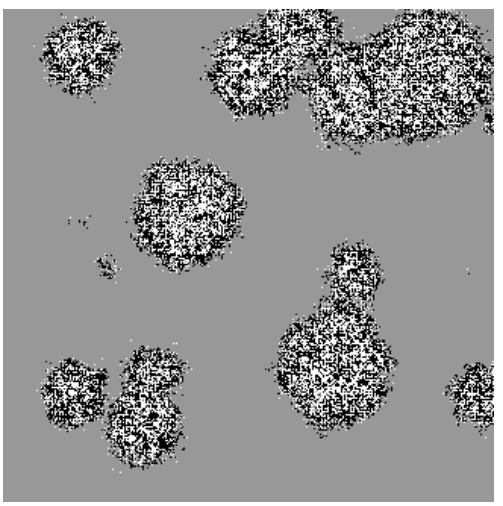

(c)

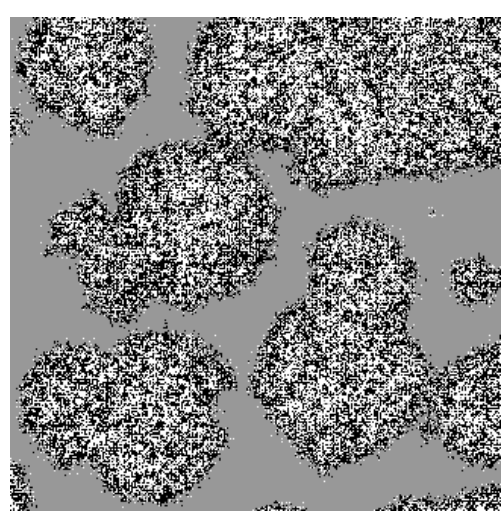

(e)

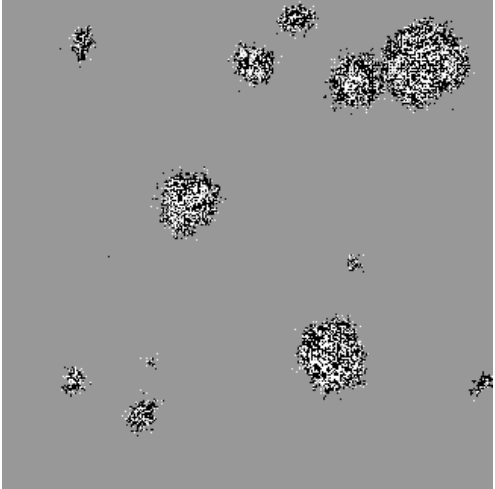

(b)

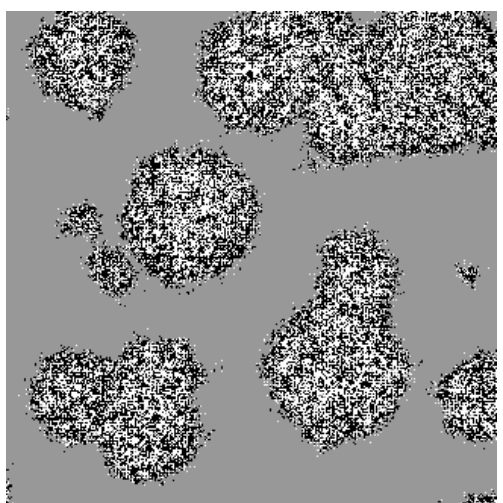

(d)

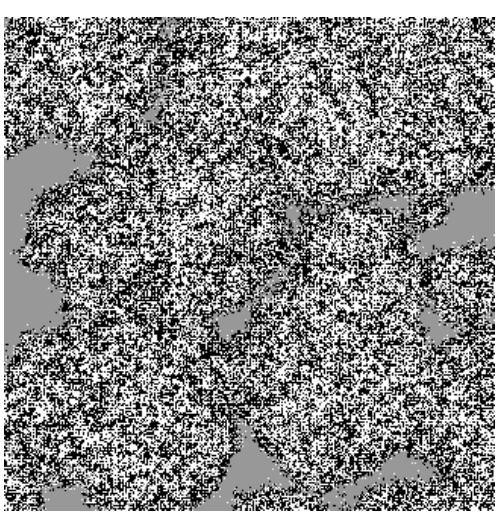

(f)

Fig. 3: Fotografías de la superficie correspondientes al espectro de la Fig. 1 cuando $W_{\text {dif }}=10 \mathrm{~s}^{-1}$. Las áreas grises indican moléculas de CO y NO adsorbidas; las negras indican átomos de $\mathrm{O}$ y $\mathrm{N}$ adsorbidos; las áreas en blanco son sitios vacíos. Solo se muestra un fragmento de $512 \times 512$ sitios de la malla completa.

\section{CONCLUSIONES}

La simulación por Monte Carlo dinámico de la reacción NO+CO sobre la superficie de Pt(100) que se ha presentado y que incluye un paso alterno en la producción de $\mathrm{N}_{2}$, no considerado en estudios anteriores, describe correctamente los resultados obtenidos en los experimentos de desorción de masa de Lesley y Schmidt (1985). Se encuentra que al escoger de manera apropiada los parámetros que entran en la simulación (velocidades de difusión y desorción, principalmente) se puede obtener una concordancia cuantitativa con los experimentos reales. Además, al analizar la evolución de una monocapa de $\mathrm{NO}+\mathrm{CO}$ en función del tiempo a temperatura constante, se observa que existe una correlación de la "explosión en superficie" durante la formación de $\mathrm{CO}_{2 \text { (gas) }}$ y $\mathrm{N}_{2 \text { (gas) }}$ con la formación de patrones espacio temporales tipo célula. 


\section{AGRADECIMIENTOS}

Salomón de J. Alas agradece el apoyo posdoctoral DGAPA-UNAM y también se agradece el apoyo al proyecto CONACYT - 49968.

\section{REFERENCIAS}

Alas, S.J. y G. Zgrablich; Study of oscillations and pattern formation in the NO+CO reaction on Pt(100) surfaces through Dynamic Monte Carlo simulation: toward a realistic mode, J. Phys. Chem. B: 110, 9499-9510 (2006).

Alas, S.J. y L. Vicente; Study of the "explosive" NO+CO reaction on a Pt(100) surface by dynamic Monte Carlo simulation, J. Mol. Catal. A: Chemical: 281, 24-34 (2008).

Avalos, L.A. y otros cuatro autores; Toward a realistic model of the NO+CO reaction on rhodium surfaces, J. Mol. Catal. A: Chemical: 228, 89-95 (2005).

Avalos, L.A. y otros cuatro autores; Dynamic Monte Carlo simulation of the NO+CO reaction on Rh(111): J. Phys. Chem. B. 110, 24964-24971 (2006).

Egelhoff, Jr. W.C.; in Chemical Physics of Solid Surfaces and Heterogeneous Catalysis, D. A. King y D. P. Woodruff, Eds.; Elsevier, Amsterdam, Vol. 4, 397-426 (1982).

Fink, Th. y otros cuatro autores; The mechanism of the "explosive" NO+CO reaction on Pt(100): experiments and mathematical modeling, Surf. Sci.: 245, 96-110 (1991a).

Fink, Th., J.P. Dath, R. Imbihl y G. Ertl; Kinetic oscillations in the NO+CO reaction on Pt(100): experiments and mathematical modeling, J. Chem. Phys.: 95, 2109-2126 (1991b).

Imbihl, R. y G. Ertl; Oscillatory kinetics in heterogeneous catalysis, Chem. Rev.: 95, 697-733 (1995).

Lesley, M.W. y L.D. Schmidt; The NO+CO reaction on Pt(100), Surf. Sci.:155, 215-240 (1985).

Lombardo, S.J., T. Fink y R. Imbihl; Simulations of the $\mathrm{NO}+\mathrm{NH}_{3}$ and $\mathrm{NO}+\mathrm{H}_{2}$ reactions on $\mathrm{Pt}(100)$ : steady state and oscillatory kinetics, J. Chem. Phys.: 98, 5526 (1993).

Makeev, A.G. y B.E. Nieuwenhuys; Mathematical modeling of the $\mathrm{NO}+\mathrm{H}_{2}$ reaction: "surface explosion", kinetic oscillations, and chaos: 108, 3740-3749 (1998).

Neurock, M., S. A. Wasileski y D. Mei; From first principles to catalytic performance: tracking molecular transformations, Chem. Eng. Sci.: 59, 4703-4714 (2004).

Ohno, Y. y otros cinco autores; Product desorption dynamics in explosive $\mathrm{NO}+\mathrm{CO}$ reaction on Pt(100), Chem. Phys. Lett.: 373, 161-166 (2003).

Perera, A. y L. Vicente; Simulation of the NO+CO oscillatory reaction on reconstructed Pt(100), Phys. Chem. Chem. Phys.: 5, 1888-1896 (2002).

Siera, J., P. Cobden, K. Tanaka y B.E. Nieuwenhuys; The NO- $\mathrm{H}_{2}$ reaction over Pt(100), oscillatory behaviour of activity and selectivity, Catal. Lett.: 10, 335-342 (1991).

Slinko, M. y otros seis autores; The $\mathrm{NO}-\mathrm{H}_{2}$ reaction over Pt(100): steady state and oscillatory kinetics, Surf. Sci.: 264, 157-170 (1992).

Zhdanov V.P.; Monte Carlo simulations of oscillations, chaos and pattern formation in heterogeneous catalytic reactions, Surf. Sci. Rep.: 45, 231-326 (2002). 